\title{
Dominio afectivo de los estudiantes de educación media hacia las matemáticas
}

\author{
Affective domain of middle school students towards mathematics
}

Domínio afetivo de estudantes do ensino médio para a matemática

\footnotetext{
Lizeth Camila Duarte-Sepúlveda ${ }^{\mathrm{a} *}$, Nataly Ricardo-Quiñones ${ }^{\mathrm{b}}$, Laura Vanessa Santos-López ${ }^{\mathrm{c} *}$

${ }^{\text {a } L i c e n c i a d a ~ e n ~ M a t e m a ́ t i c a s, ~ l i z e t h c a m i l a d s @ u f p s . e d u . c o, ~ O R C I D ~ 0000-0002-9486-2765, ~ U n i v e r s i d a d ~ F r a n c i s c o ~ d e ~ P a u l a ~ S a n t a n d e r, ~ C u ́ c u t a, ~ C o l o m b i a . ~}$

b Licenciada en Matemáticas, natalyrq@ufps.edu.co, ORCID 0000-0002-1475-2161, Universidad Francisco de Paula Santander, Cúcuta, Colombia.

$\mathbf{c}^{*}$ Licenciada en Matemáticas, lauravanessasl@ufps.edu.co, ORCID 0000-0002-4686-9700, Universidad Francisco de Paula Santander, Cúcuta, Colombia.
}

Forma de citar: Duarte, L. C., Ricardo, N. \& Santos, L. V. (2018). Dominio afectivo de los estudiantes de educación media hacia las matemáticas. Perspectivas, 3(2), 60-71.

Recibido: Enero 25 de 2018

Aceptado: Junio 10 de 2018

\section{Palabras clave}

Actitud

Creencia

Dominio Afectivo

Emoción
Resumen: Las matemáticas son fundamentales en el currículo escolar, siendo una materia obligatoria para todos los niveles educativos, que contribuye al desarrollo del pensamiento lógico y el pensamiento matemático de los estudiantes. Por esa razón, se plantea relacionar el dominio afectivo que tienen los estudiantes de educación media hacia las matemáticas con el rendimiento académico en el área. Para ello, primero se determina las creencias de los estudiantes de educación media hacía las matemáticas. Seguido de, describir las emociones que poseen los estudiantes de educación media hacia las matemáticas. Para finalmente, identificar las actitudes que presentan los estudiantes de educación media hacia las matemáticas. El trabajo se realiza en un tiempo de seis meses, en la Institución Educativa Misael Pastrana Borrero de Cúcuta, Norte de Santander. La investigación es de tipo correlativa. La población para desarrollar el proyecto se tuvo en cuenta los estudiantes del Colegio Misael Pastrana Borrero pertenecientes a la sede central, el cual ofrece el servicio educativo de sexto a undécimo grado en jornada única y está conformada por 800 estudiantes. En esta investigación la muestra será tomada por conveniencia, puesto que no

\footnotetext{
*Autor para correspondencia

Laura Vanessa Santos López

lauravanessasl@ufps.edu.co
} 
se realiza un muestreo probabilístico sino, que será aplicada a todos los estudiantes de décimo y undécimo grado de la sede central Misael Pastrana Borrero. Se recolecta información a partir de un cuestionario sometido a juicio de expertos y adaptado al contexto para tal fin. Los datos son procesados a través de herramientas de Microsoft office y el programa SPSS. La información obtenida establece que el dominio afectivo que poseen los estudiantes hacia las matemáticas es positivo y su desempeño académico fue bueno, además se encuentra que existe una posible relación en algunos aspectos del dominio afectivo con el rendimiento académico de los estudiantes.

\section{Keywords}

Attitude

Belief

Dominion Affective

Emotion

\section{Palavras chave}

Atitude

Crença
Abstract: Mathematics is fundamental in the school curriculum, being a compulsory subject for all educational levels, which contributes to the development of logical thinking and mathematical thinking of students. For this reason, it is proposed to relate the affective domain that middle school students have to mathematics with academic performance in the area. To do this, the beliefs of the students of secondary education towards mathematics are first determined. Followed by, describe the emotions that middle school students have towards mathematics. To finally, identify the attitudes that middle school students present towards mathematics. The work is carried out in a period of six months, at the Misael Pastrana Borrero Educational Institution in Cúcuta, Norte de Santander. The investigation is of correlative type. The population to develop the project was taken into account the students of the Misael Pastrana Borrero School belonging to the central headquarters, which offers the educational service from sixth to eleventh grade in a single day and is made up of 800 students. In this investigation the sample will be taken for convenience, since probabilistic sampling is not done but, it will be applied to all students of tenth and eleventh grade of the Misael Pastrana Borrero headquarters. Information is collected from a questionnaire submitted to expert judgment and adapted to the context for that purpose. The data is processed through Microsoft Office tools and the SPSS program. The information obtained establishes that the affective domain that the students have towards mathematics is positive and their academic performance was good, also it is found that there is a possible relationship in some aspects of the affective domain with the academic performance of the students.

Resumo: A matemática é fundamental no currículo escolar, sendo uma disciplina obrigatória para todos os níveis de ensino, o que contribui para o desenvolvimento do pensamento lógico e do pensamento matemático dos alunos. Por essa razão, propõe-se relacionar o domínio afetivo que os alunos do ensino médio têm à matemática com o desempenho 
Domínio Afetivo

Emoção

acadêmico na área. Para fazer isso, as crenças dos alunos do ensino médio em relação à matemática são determinadas pela primeira vez. Seguido por, descreva as emoções que os alunos do ensino médio têm em relação à matemática. Por fim, identificar as atitudes que os alunos do ensino médio apresentam em relação à matemática. $\mathrm{O}$ trabalho é realizado em um período de seis meses, na Instituição Educacional Misael Pastrana Borrero, em Cúcuta, Norte de Santander. A investigação é do tipo correlativo. População para desenvolver o projeto levou em conta os alunos Misael Pastrana escola pertencente à sede, que fornece serviços educacionais em sexto através os dias décimo primeiro grau e é composta de 800 alunos. Nesta investigação a amostra será retirada por conveniência, uma vez que a amostragem probabilística não é feita, mas será aplicada a todos os alunos de décimo e décimo primeiro ano da sede do Misael Pastrana Borrero. As informações são coletadas de um questionário submetido a julgamento de especialistas e adaptadas ao contexto para essa finalidade. Os dados são processados através das ferramentas do Microsoft Office e do programa SPSS. As informações

\section{Introducción}

Es reconocido que las matemáticas son útiles y necesarias para desenvolverse adecuadamente en un determinado contexto (Penagos, Mariño, \& Hernández, 2017); sin embargo, se evidencian dificultades en el aprendizaje de esta disciplina, tal como se muestra en los resultados de las pruebas de estado (Fernándes, 2009; Velásquez-Luna, CelisGutiérrez \& Hernández-Suárez, 2017; HernándezSuarez, Prada-Núñez \& Ramírez-Leal, 2017); dichas dificultades han sido objeto de estudio de diversas investigaciones, varias de ellas dan a conocer que las cuestiones afectivas tienen un papel importante en el proceso de enseñanza - aprendizaje; es decir, la problemática no se debe únicamente a la naturaleza de la matemática sino también a una serie de factores afectivos, los cuales podrían relacionarse con el rendimiento académico en matemáticas de los estudiantes (Gil, Blanco \& Guerrero, 2006).

En esta investigación se analizará la influencia que el dominio afectivo (creencias, actitudes y emociones) ejerce en el aprendizaje de las matemáticas y cómo los afectos van a condicionar el éxito y fracaso del estudiantado a la hora de enfrentarse a esta disciplina, siguiendo lo expresado por Gómez (citado en Gil, Blanco \& Guerrero, 2005) en el aprendizaje de las matemáticas se observa gran cantidad de fracasos en los diferentes niveles educativos, los cuales se atribuyen a una serie de factores que pueden generar actitudes negativas, siendo primordial identificar dichas actitudes para disminuir el efecto que producen. Una revisión de antecedentes sobre el dominio el dominio afectivo se encuentra en el trabajo de Fernández, Hernández, Prada y Ramírez (2018).

De ahí se considera necesario el estudio del dominio afectivo de los estudiantes hacia las matemáticas, por lo que permite identificar las creencias, emociones y actitudes de estos hacia la materia con el objetivo de relacionarlas con el rendimiento académico, cuyo propósito es reconocer cómo influye el dominio afectivo en el rendimiento académico de los estudiantes de educación media de la Institución Educativa Misael Pastrana Borrero, para así tomar medidas correctivas en la metodología y didáctica empleada en las clases de matemáticas y ayudar a mejorar el componente afectivo.

Además, la importancia del aprendizaje de las matemáticas, no es exclusivo para adquirir 
conocimiento matemático sino que también sirve como base para el aprendizaje de otras asignaturas, dado que analizar y tratar información son habilidades que se desarrollan a través de la matemática, las cuales son necesarias en todo profesional; por lo tanto, como docentes se deben atender las habilidades que lleven a los estudiantes a la búsqueda y creación de conocimiento y reconocer que los estudiantes no son únicamente un recipiente vacío que se llena de conocimiento sino también tienen una parte afectiva que puede influir en su comportamiento Goleman (citado por Benito, 2014).

Hidalgo, Maroto \& Palacios (2004), establecen que las dificultades en el aprendizaje matemático son acumulativas; es decir, los problemas que tienen los estudiantes en básica primaria se heredan en secundaria y los de secundaria afectan en la educación superior y además el rechazo hacia las matemáticas no "nace" en el estudiante sino se "hace" y depende del nivel de complejidad de cada grado.

Caballero, Blanco, \& Guerrero (2007), manifiestan que las matemáticas son útiles y necesarias para la vida, permiten asimilar y dominar otras asignaturas relacionadas con ella; también es una materia memorística y mecánica donde el conocimiento de fórmulas, reglas o procedimientos facilita generalmente la resolución de los problemas; en cuanto a los estudiantes como aprendices de matemáticas, señalan que estos buscan diversas maneras y métodos para resolver problemas y quien no lo hace termina estudiando de memoria; además expresan que el estudiantado atribuye el éxito en matemáticas a la actitud del profesor, aunque estos no empleen variedad de medios y ejemplos que permitan relacionar las matemáticas con la vida diaria y valoran la claridad, simpatía, entusiasmo, disponibilidad, cercanía e interés que presentan en las clases; de igual manera establecen que las expectativas positivas de los padres hacia los estudiantes generan buenos resultados de estos en la materia; se evidencia que generalmente los estudiantes sienten curiosidad por la solución de problemas obteniendo gran placer cuando tienen éxito y una sensación de fracaso cuando no y se encontró que la perseverancia, esfuerzo y paciencia son fundamentales para la resolución de problemas; finalmente en la formación de los estudios de magisterio se produjo un cambio favorable en la percepción y valoración otorgada a las matemáticas.

Gil et al. (2006), señalan que los hombres se sienten más capacitados en matemáticas; sin embargo, su desempeño es ligeramente superior al de las mujeres; también se refleja que el género masculino demuestra tener más seguridad, confianza y tranquilidad al momento de enfrentarse al proceso de resolución de problemas; mientras las mujeres son más propensas a dejarse influenciar por el gusto o disgusto que tienen hacia la materia a la hora de elegir una modalidad de bachillerato; además se encontró que para los hombres la actitud del docente y la suerte no influye en su rendimiento académico, pero en las mujeres sí afecta; de ahí se obtuvo que los estudiantes (hombres) tienen un mejor autoconcepto matemático que las estudiantes (mujeres) y se demuestra que existe una relación entre el rendimiento académico y el auto concepto que tiene el alumno sobre sí mismo; sin embargo, esto no permite establecer una relación directa entre el género y el autoconcepto matemático puesto que la brecha entre el género del alumnado en cada una de las variables estudiadas es ligeramente inferior.

Noguera, RamírezyMartínez(2016), evidencian que los estudiantes sienten predisposición frente a la enseñanza de las matemáticas y la asistencia del docente; a su vez, tienen un comportamiento inadecuado cuando el docente desarrolla la clase, el cual cambia cuando el docente toma represarías y atemoriza con un determinado castigo; por lo tanto, no se sienten motivados en el aprendizaje de las matemáticas y se refleja en su poca participación en clase, mostrándose perezosos. Se muestra que los estudiantes tienen dificultades cuando se enfrentan a problemas matemáticos, afirman que cada vez les gusta menos las matemáticas considerando que no son importantes y por más que se esfuerzan siempre obtienen un bajo rendimiento en la materia. Al correlacionar las actitudes y el rendimiento 
académico se encontró que entre más actitudes negativas sobre las matemáticas, mayor deficiencia en el rendimiento académico, así mismo entre más actitudes favorables hacia las matemáticas, mejor rendimiento académico.

Dentro del desarrollo de la investigación se hizo necesario aclarar ciertas definiciones que permitieron orientar el propósito de la investigación para evitar caer en ambigüedades. En este orden de ideas, se propusieron los siguientes conceptos:

EI dominio afectivo: Es definido por McLeod (citado por Gil et al, 2006) como "un extenso rango de sentimientos y humores (estados de ánimo) que son generalmente considerados como algo diferente a la pura cognición" (p. 16). Donde las actitudes, creencias y emociones son sus componentes principales.

La actitud: Es considerada como una evaluación (positiva, negativa) constante que realizan las personas sobre un objeto de actitud tales como personas, ideas, situaciones, objetos lo cual influye en su comportamiento Hart (citado por Caballero et al, 2007).

Para Clemente (citado por Martínez, 2008) las actitudes son sentimientos positivos o negativos sobre un objeto o situaciones lo cual va a condicionar su pensamiento y forma de actuar. "las actitudes son instancias que predisponen y dirigen al sujeto sobre hechos de la realidad, filtran las percepciones y orientan el pensamiento para adaptarlo al contexto". Es decir, son predisposiciones positivas o negativas que el sujeto opta, cambiando su manera de pensar e influyendo en su comportamiento.

De acuerdo a lo planteado hasta ahora, se puede decir que las actitudes son predisposición (positiva, negativa) que opta el sujeto frente a un objeto o situación y que condiciona su comportamiento.

Las actitudes se manifiestan a través de ideas, percepciones, gustos, preferencias, opiniones, creencias, emociones, sentimientos, tendencia a actuar o comportamientos y estos se dividen desde la perspectiva psicosocial en tres componentes (Martínez, 2005):
Componente cognitivo (el saber): Es la información y creencias que tiene el sujeto sobre su objeto de actitud.

Componente afectivo (el sentir): Son el conjunto de sentimientos y emociones que presenta el sujeto frente al objeto de actitud.

\section{Componente conductual}

(el comportamiento): La forma de actuar frente al objeto de actitud.

Las creencias: las creencias son verdades personales, que se construyen en un proceso de formación lo cual influye en el comportamiento y acciones específicas (Martínez, 2005). Con relación a las matemáticas las creencias son verdades personales que se adquieren por la experiencia sobre la enseñanza y aprendizaje matemático Gilbert (citado por Carrasco, et al, 2007).

Para Hidalgo et al, (2004) las creencias matemáticas son los pensamientos personales que se tienen sobre la disciplina, su enseñanza y aprendizaje que son creadas por el individuo a través de la experiencia.

McLeod (citado por Gil et al, 2005) propone una clasificación de las creencias de los estudiantes de acuerdo al objeto de creencia:

Creencias hacia las matemáticas y su aprendizaje.

Creencias sobre uno mismo como aprendiz de matemáticas.

Creencias sobre la enseñanza de las matemáticas.

Creencias sobre el contexto social.

Las emociones: son un conjunto de sentimientos que se pueden observar directamente en los individuos por producir alteraciones fisiológicas, como sudoración, respiración alterada, enrojecimiento facial (Martínez, 2005).

Las reacciones emocionales tienen una influencia en el aprendizaje, es decir si se experimenta un sentimiento de ira o miedo en el aprendizaje esto va obstaculizar al estudiante y a su capacidad de aprender (Velazco citado por Martínez, 2005).

Para Olguín (citado por Martínez, 2005) las emociones se conforman por tres componentes: 
El perceptivo: Destinado a la detección de estímulos. Incluye elementos hereditarios y también fruto de las experiencias del sujeto.

El motivacional: Encargado de impulsar, mantener y dirigir la conducta de los sujetos hacia determinados objetos.

El conductual: Depende de tres manifestaciones:(a) la reacción fisiológica perceptible, (b) los pensamientos, y (c) la conducta manifiesta.

Como se ha mencionado anteriormente el dominio afectivo está conformado por tres componentes, que son las actitudes, creencias y emociones (Estrada citado por Chaves, Castillo \& Gamboa, 2008) menciona que hay una relación cíclica entre ellos, es decir cuando el estudiante se enfrenta al aprendizaje matemático ya tiene unas creencias sobre las matemáticas y sobre el mismo, durante este proceso el estudiante recibe estímulos que harán que reaccione emocionalmente ya sea de forma positiva o negativa, estas reacciones generan sentimientos (emociones) de frustración o de satisfacción y si esta situación ocurre constantemente estos sentimiento se consolidad hasta convertirse en actitudes positivas o negativas hacia la matemáticas, su aprendizaje y enseñanza.

\section{Materiales y métodos}

\section{Naturaleza de estudio}

En la presente investigación se pretende adquirir las opiniones de los estudiantes en relación al tema de estudio y conocer su rendimiento académico, para posteriormente hacer una interpretación de cada uno y relacionarlos. Por tanto, la investigación se enmarca dentro de la metodología de tipo correlativo puesto que tienen el propósito de conocer el comportamiento que adquiere una variable a partir del comportamiento de las otras variables (Hernández, Fernández \& Baptista, 2003) y está sustentada bajo el enfoque investigativo cuantitativo, el cual permite probar la hipótesis a través de la medición numérica y análisis estadísticos, para así establecer patrones de comportamientos y probar las teorías (Hernández, Fernández \& Baptista, 2010).

La ejecución de esta propuesta investigativa consta dela aplicación de un cuestionarioa estudiantes de educación media, sobre temas relacionados con las actitudes, emociones y creencias que tiene sobre las matemáticas, las cuales serán analizadas, para así interpretar cuál es su relación con el rendimiento académico en el área.

\section{Muestra}

Para desarrollar el proyecto se tuvo en cuenta los estudiantes del Colegio Misael Pastrana Borrero pertenecientes a la sede central, el cual ofrece el servicio educativo de sexto a undécimo grado en jornada única, se tomará una muestra por conveniencia, puesto que no se realiza un muestreo probabilístico sino que será aplicada a todos los estudiantes de décimo y undécimo grado conformada por 171 estudiantes de educación media.

\section{Recolección de información}

Para la recolección de los datos se aplicó la técnica Escala Likert, la cual tiene como finalidad medir actitudes a través de una serie de preguntas referentes a un tema específico donde el encuestado expresa su grado de acuerdo o desacuerdo, teniendo comúnmente como alternativa de respuestas cinco categorías (García, Aguilera \& Castillo. 2011). Concretamente se empleó el cuestionario como instrumento, el cual según Tamayo (citado por Sánchez, 2010) está formado por una serie de preguntas que se consideran relevantes para determinar las características de las variables que son objeto de estudio tomando el cuestionario sobre creencias, emociones y actitudes hacia las matemáticas elaborado por Gil et al, (2006) el cual fue adaptado a los estudiantes de educación media de la Institución Educativa Misael Pastrana Borrero. 


\section{Técnicas de procesamiento y análisis de la información}

Antes de empezar a hacer cualquier análisis estadístico se procede a revisar cada uno de los enunciados de todos los bloques con el fin de garantizar que cada uno de ellos estén expresados de manera afirmativa y positiva, para evitar que vayan a dar en contra vía las afirmaciones.

Para realizar la correlación se trabajaron las calificaciones de los estudiantes de acuerdo a lo establecido por el MEN en el Decreto 1290 de 2009 en el cual se define la escala de valoración nacional de los desempeños de los estudiantes que deben adoptar cada institución educativa en cuatro niveles de desempeño: superior, alto básico y bajo.

La información recolectada fue procesada a través de herramientas de Microsoft Excel donde se organizan los datos en cinco bloques y se calcula el promedio de cada uno de los ítems, además se analiza a través del software estadístico SPSS 21 utilizando la técnica "Prueba Chi-Cuadrado", la cual permite analizar la independencia o posible correlación entre los ítems de cada bloque y el rendimiento académico.

\section{Resultados y discusiones}

Inicialmente se describe las principales características del objeto de estudio donde se tiene en cuenta las creencias, actitudes y emociones de los estudiantes de educación media hacia las matemáticas y su relación con el rendimiento académico.

En la tabla 1 se encuentran las creencias sobre la materia, donde la mayoría de los estudiantes consideran que al solucionar un problema matemático es más importante el proceso que se utiliza que el mismo resultado, mostrando la matemática como una materia sistemática. En cuanto al aprendizaje de matemáticas, se encuentran opiniones muy similares entre los estudiantes que piensan que se aprende mejor los conceptos matemáticos cuando se estudia en grupo que cuando se estudia de forma individual, contra los que consideran que se obtiene un mejor aprendizaje cuando se estudia individualmente; sin embargo, existe una ligera inclinación hacia el trabajo grupal. Además, los estudiantes creen que para dominar ésta asignatura es esencial aprenderse de memoria los conceptos y fórmulas dados por el docente, considerando la memorización como el método más eficaz para poder desarrollar o solucionar problemas matemáticos; asumiendo a la matemática como un área importante y necesaria en su formación, la cual es de utilidad en su diario vivir.

Tabla 1. Bloque 1 creencias de la materia

\begin{tabular}{|c|c|c|c|}
\hline & Creencias de la materia & Promedio & $\begin{array}{l}\text { Valor Chi- } \\
\text { cuadrado }\end{array}$ \\
\hline 1 & Las matemáticas son útiles y necesarias en todos los ámbitos de la vida. & 0,021 & 3,30 \\
\hline 2 & Las matemáticas son fáciles, motivantes y permiten resolver situaciones de la vida real. & 0,000 & 2,91 \\
\hline 3 & En matemáticas es fundamental aprenderse de memoria los conceptos, formulas y reglas. & 0,881 & 3,34 \\
\hline 4 & $\begin{array}{l}\text { Generalmente los problemas de matemáticas se resuelven en pocos minutos si se conoce la formula, que ha } \\
\text { explicado el profesor }\end{array}$ & 0,873 & 3,30 \\
\hline 5 & Me interesan todos los temas así no sean evaluados porque todos son importantes y los que tengo que conocer. & 0,446 & 2,72 \\
\hline 6 & La matemática se aprende mejor cuando se estudia de forma individual que de manera grupal. & 0,454 & 2,41 \\
\hline 7 & Cuando resuelvo un problema de matemáticas es más importante el resultado que el proceso utilizado. & 0,507 & 2,17 \\
\hline 8 & $\begin{array}{l}\text { Sabiendo resolver los problemas que propone el profesor en clase, es posible solucionar otros del mismo tipo si } \\
\text { solo les han cambiado los datos. }\end{array}$ & 0,323 & 3,25 \\
\hline 9 & $\begin{array}{c}\text { Las destrezas o habilidades que utilizo en clase para resolver problemas tienen relación con las que utilizo } \\
\text { para resolver problemas en la vida cotidiana. }\end{array}$ & 2,58 & 0,570 \\
\hline 10 & Busco distintos métodos para resolver un problema. & 3,13 & 0,313 \\
\hline 11 & Aprendo más cuando formulo nuevos problemas & 3,09 & 0,450 \\
\hline
\end{tabular}


Asimismo, se consideran capaces de resolver problemas si ya los han trabajado con anterioridad y la creación de nuevos problemas les permite obtener más aprendizaje, asumiendo que existen diferentes métodos o caminos para hallar la solución de ellos y las habilidades que utilizan en clase les son útiles para resolver situaciones de su vida diaria. Respecto al nivel de dificultad de las matemáticas, piensan que es una materia fácil y motivante, considerando que el área no está alejada de la realidad y que todos los temas son fundamentales sin importar si son o no evaluados.

A partir de los datos obtenidos de la muestra se permite verificar que los ítems 1 y 2 produjeron p-valores menores al nivel de significancia lo que es equivalente a decir que existe relación entre el rendimiento académico y la importancia de la matemática en la vida diaria, a su vez presenta una relación con el nivel de dificultad y utilidad de las matemáticas.

Tabla 2. Bloque 2 creencias sobre uno mismo

\begin{tabular}{|c|c|c|c|}
\hline & Creencias sobre uno mismo & Promedio & $\begin{array}{l}\text { Valor Chi- } \\
\text { cuadrado }\end{array}$ \\
\hline 12 & $\begin{array}{l}\text { A la hora de escoger una determinada modalidad, me dejo llevar por el gusto o disgusto que tengo hacia la } \\
\text { matemática. }\end{array}$ & 0,781 & 2,34 \\
\hline 13 & $\begin{array}{c}\text { El ser buen estudiante en matemáticas (sacar buenas notas, tener buena actitud) te hace sentir más valorado y } \\
\text { admirado por tus compañeros. }\end{array}$ & 0,061 & 2,82 \\
\hline 14 & $\begin{array}{c}\text { Si comprendo las matemáticas, podré entender y dominar fácilmente otras asignaturas relacionadas con } \\
\text { ellas (como física, química, etc.) }\end{array}$ & 0,377 & 2,17 \\
\hline 15 & Mi rendimiento en matemáticas depende en gran medida de la actitud del profesor(a) hacia mí. & 0,741 & 2,32 \\
\hline 16 & $\begin{array}{c}\text { Cuando dedico más tiempo de estudio a las matemáticas obtengo mejores resultados en la resolución de } \\
\text { problemas. }\end{array}$ & 0,295 & 3,25 \\
\hline 17 & Cuando resuelvo un problema estoy seguro que el resultado es correcto. & 0,051 & 2,14 \\
\hline 18 & Tengo confianza en mí mismo cuando me enfrento a los problemas de matemáticas. & 0,021 & 2,81 \\
\hline 19 & Me considero muy capaz y hábil en matemáticas. & 0,040 & 2,57 \\
\hline 20 & Estoy calmado y tranquilo cuando resuelvo problemas de matemáticas. & 0,034 & 2,81 \\
\hline 21 & Cuando me esfuerzo en la resolución de un problema suelo dar con el resultado correcto. & 0,550 & 3,01 \\
\hline 22 & La suerte influye a la hora de resolver con éxito un problema de matemáticas. & 0,455 & 2,01 \\
\hline
\end{tabular}

En cuanto al bloque referido a las creencias que tiene el estudiante sobre sí mismo como aprendiz de matemáticas, los encuestados consideran que la suerte no es un factor clave para el éxito en la solución de problemas matemáticos y afirman sentirse tranquilos al momento de resolverlos demostrando que a través de la práctica y el esfuerzo se puede llegar a un mejor resultado.

Por otra parte, consideran la matemática como un área independiente y no transversal; además, el obtener buenas calificaciones hace sentir al estudiantado más valorado por sus compañeros, y este rendimiento lo que no se debe únicamente a la actitud del profesor sino que también existen otros factores que pueden influir.

Con base a los valores arrojados por la prueba chi-cuadrado se puede corroborar que tanto la confianza en sí mismo a la hora de resolver un problema; la capacidad y habilidad en matemáticas como la calma y tranquilidad cuando se resuelven problemas matemáticos se relacionan con el rendimiento académico. 
Tabla 3. Bloque 3 creencias sobre el profesor

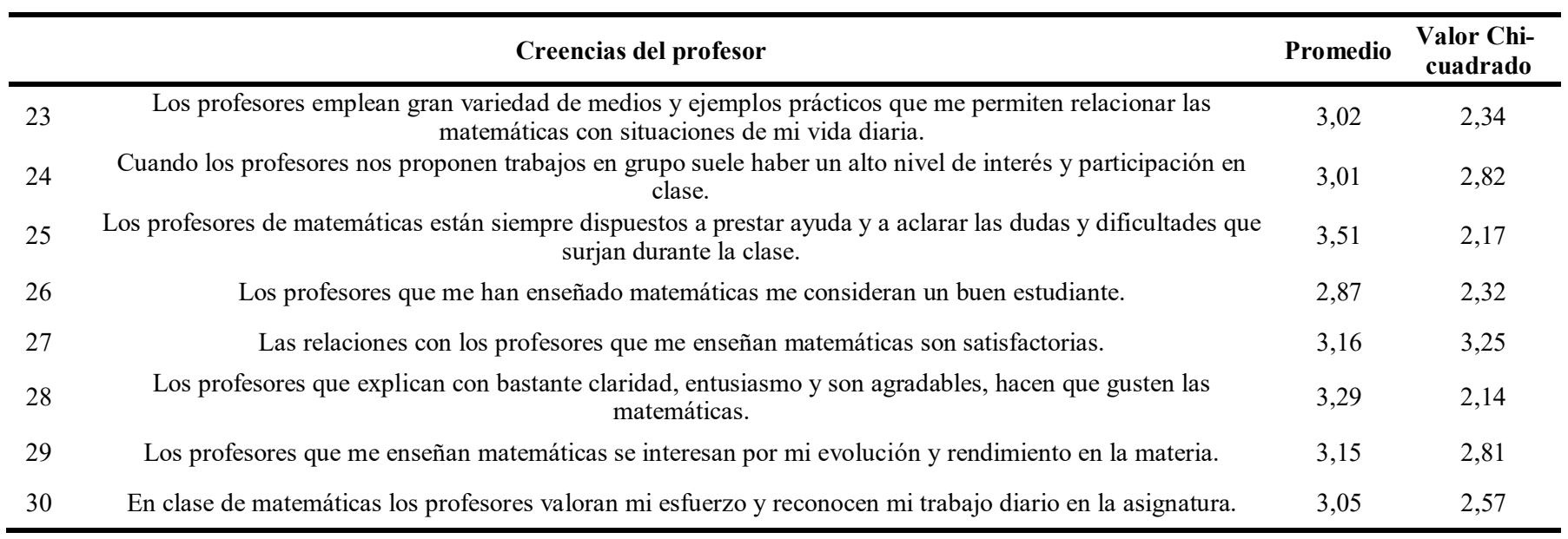

A partir de la tabla 3, se evidencia que la mayor parte de los encuestados son considerados buenos estudiantes por parte de los profesores que les han enseñado matemáticas. Además, presentan un alto nivel de interés y participación en clase cuando los profesores ponen trabajos en grupo, así mismo los profesores emplean diferentes medios y ejemplos prácticos que permiten relacionar las matemáticas con diferentes situaciones de su vida diaria, lo cual les ayuda a entender para qué les sirven y cómo las pueden utilizar en diferentes contextos.

También, se observa que el profesor se interesa por la evolución y el rendimiento del estudiante en el área, valorando el esfuerzo y trabajo diario que éste realiza; mostrándose dispuesto a ayudar, aclarar dudas y dificultades que se presentan, de ahí que el hecho de realizar las clases con claridad, entusiasmo y actitud agradable, hace que se despierte un gusto por la materia creando entre ellos relaciones interpersonales satisfactorias.

De acuerdo en la información suministrada se confirma que la disposición que tiene el docente en solucionar dificultades y su opinión sobre el estudiante influye en el rendimiento académico.

Tabla 4. Bloque 4 creencias de la sociedad

\begin{tabular}{lccc}
\hline & Creencias de la sociedad & Promedio & $\begin{array}{c}\text { Valor Chi- } \\
\text { cuadrado }\end{array}$ \\
\hline 31 & Alguno de mis padres espera de mí buenos resultados en matemáticas. & 3.32 & 0,289 \\
32 & Las matemáticas que nos enseñan en el colegio les interesan a mis padres. & 3.02 & 0,134 \\
33 & Alguno de mis padres era bastante bueno resolviendo problemas de matemáticas. & 2.85 & 0,758 \\
34 & Alguno de mis padres me anima y ayuda con los problemas de matemáticas. & 2.36 & 0,573 \\
35 & Mis amigos afrontan las matemáticas. & 2.39 & 0,412 \\
36 & Las profesiones mejor remuneradas económicamente están relacionadas con las matemáticas. & 3.16 & 0,790 \\
37 & La gente a la que le gustan las matemáticas suelen ser normales. & 2.78 & 0,333 \\
38 & El aumentar mis conocimientos matemáticos me hará sentir una persona competente en la sociedad. & 2.93 & 0,266 \\
39 & Las matemáticas son para personas con cabezas inteligentes y creativas. & 1.92 & 0,610 \\
40 & Dominar los contenidos matemáticos me permitirá tener éxito en mis estudios posteriores. & 3.24 & 0,506 \\
41 & La gente que es buena en matemáticas gasta menos tiempo pensando cómo resolver un problema. & 2.69 & 0,642 \\
\hline
\end{tabular}


De acuerdo a la información de la tabla 4, se establece que la mayoría de los estudiantes consideran que las matemáticas no son sólo para inteligentes y el hecho de gustarle la materia no lo hace una persona diferente y esto haría que empleara menos tiempo pensando cómo resolver un problema. Además, se encuentra que los padres de familia esperan de ellos un buen resultado académico en el área estando atentos a su proceso académico; sin embargo, no los animan a resolver problemas matemáticos.

Asimismo, los estudiantes creen que si se dedican al estudio y tienen buen rendimiento académico, esto les permitirá ser competentes en la sociedad y obtener éxito en sus estudios posteriores; considerando que las carreras universitarias mejor remuneradas son aquellas relacionadas con las matemáticas.

A partir de la tabla anterior se verifica que los ítems de este bloque no presentan relación con el rendimiento académico, puesto que el nivel de significancia es mayor al permitido; por lo tanto, las variables son independientes.

Tabla 5. Bloque 5 actitudes y reacciones emocionales

\begin{tabular}{lcccc}
\hline & Actitudes y reacciones emocionales & Promedio & $\begin{array}{c}\text { Valor Chi- } \\
\text { cuadrado }\end{array}$ \\
\hline 42 & Las clases de matemáticas se me hacen amenas, ligeras, me siento a gusto y siento deseos de permanecer en & 2.86 & 0,001 \\
ellas. & 2.92 & 0,000 \\
43 & Disfruto los días que tenemos clases de matemáticas porque me interesan y me atraen. & 2.93 & 0,129 \\
44 & Ante un problema complicado suelo intentar resolverlo hasta el final. & 3.07 & 0,866 \\
45 & Cuando me enfrento a un problema experimento mucha curiosidad por conocer la solución. & 2.36 & 0,004 \\
46 & Me animo y siento tranquilidad cuando el profesor me propone (por sorpresa) que resuelva un problema. & 2.91 & 0,105 \\
47 & Cuando resuelvo problemas en grupo tengo más seguridad en mí mismo. & 2.28 & 0,416 \\
48 & Cuando descifro la resolución de un problema empiezo a sentirme seguro, esperanzado y tranquilo. & 2.48 & 0,505 \\
49 & Si encuentro la solución de un problema tengo la sensación de haber triunfado y aprovechado el tiempo. & 3.44 & 0,133 \\
50 & Me provoca gran satisfacción llegar a resolver con éxito un problema matemático. & 2.98 & 0,156 \\
51 & Cuando fracaso al resolver un problema matemático lo intento nuevamente. & 3.40 & 0,148 \\
52 & La resolución de un problema exige esfuerzo, perseverancia y paciencia. & & \\
\hline
\end{tabular}

La información obtenida en la tabla 5 revela que el estudiantado al enfrentarse a un problema y no encontrar fácilmente su solución siente frustración y pierde la confianza sobre sí mismo, es por ello, que al no llegar a la correcta solución del problema tienen la sensación de haber perdido el tiempo y de haber fracasado en su tarea de estudiante.

Por otra parte, afirman sentirse inseguros e intranquilos cuando el profesor les propone por sorpresa un problema, pero experimentan curiosidad por conocer el resultado, asumiendo que el esfuerzo y la perseverancia son necesarios para el éxito en la solución del mismo, disfrutando el instante en llegar a la correcta solución; por ello, al momento de realizar un problema con alto nivel de dificultad el estudiantado suele insistir en hallar su solución, al no encontrarla persisten en resolver el problema y no abandonan fácilmente su desarrollo.

Con relación a las clases de matemáticas los estudiantes disfrutan los días que tienen clase porque les interesan, se sienten a gusto y les atraen, sintiendo seguridad y confianza en sí mismos al momento de trabajar en grupo. 
En consecuencia a los resultados de la prueba chi-cuadrado se corrobora que existe relación entre el rendimiento académico y las emociones generadas al resolver un problema y al mismo tiempo con el gusto por las clases de matemáticas.

\section{Conclusiones}

Con el desarrollo de la presente investigación se encontró el dominio afectivo de los estudiantes de educación media hacia las matemáticas y su relación con el rendimiento académico, del cual se puede inferir que los estudiantes presentan un dominio afectivo positivo hacia las matemáticas, puesto que consideran que aunque sea un área memorística es necesaria para ser competente socialmente, por lo tanto se esfuerzan en aprenderla y no se dejan influenciar por opiniones negativas hacia ella, aceptando que la actitud y la metodología del docente influye en su gusto por la materia.

Además se confirma la teoría de la discrepancia de Mandler (citado por Gómez, 1997) puesto que los estudiantes afirman que al momento de enfrentarse a problemas matemáticos y presentar un bloqueo en la solución del mismo suelen sentirse inseguros, desesperados y nerviosos y cuando llegan al resultado dudan si este es correcto, pero al realizar la interpretación de estas emociones establecen que el esfuerzo, la paciencia, tranquilidad y perseverancia es lo que les permitirá tener éxito en sus estudios y hace que en un futuro los estudiantes estén preparados y preparen el organismo para una repuesta más efectiva.

Finalmente se obtiene que tanto el rendimiento académico como el dominio afectivo de los estudiantes son positivos, sin embargo; los resultados no permiten establecer una relación directa entre las variables; pero se encuentra que existe una posible relación entre algunos aspectos del dominio afectivo y el rendimiento académico; de ahí se puede concluir de acuerdo a la teoría de la atribución de Weiner (citado en Vázquez y Manassero, 1989) que estos aspectos son las principales causas del rendimiento académico que presentan los estudiantes de 10 y 11, es decir; la importancia de la matemática para la vida, la confianza propia, las emociones al enfrentarse a problemas, el concepto que tiene el docente sobre estudiante y la actitud del docente son las atribuciones causales del rendimiento académico obtenido durante el primer periodo.

\section{Agradecimientos}

Primeramente agradecer a Dios por hacer esto posible, a nuestros padres por su esfuerzo y apoyo incondicional en nuestro proceso de formación y a nuestros profesores por cada uno de sus consejos para crecer personal y profesionalmente.

\section{Referencias}

Alonso, A. V., \& Mas, M. M. (1986). La teoría de la atribución y el rendimiento escolar. Educación y cultura: revista mallorquina de pedagogía, (7), 225-241.

Alonso, S., Sáez, A \& Picos, A. (2004). ¿Por qué se rechazan las matemáticas? Análisis evolutivo y multivariante de actitudes relevantes hacia las matemáticas. Revista de educación, 1(334), pp. 75-95.

Benito Álvarez, P. (2014). Los factores afectivos en la enseñanza y aprendizaje de lenguas extranjeras.

Caballero, A., Blanco, L. J. y Guerrero, E. (2007), Las actitudes y emociones ante las Matemáticas de los estudiantes para Maestros de la Facultad de Educación de la Universidad de Extremadura.

Chacón, I. M. G., \& Hoz, A. D. L. O. (1997). Procesos de aprendizaje en matemáticas con poblaciones de fracaso escolar en contextos de exclusión social. Las influencias afectivas en el conocimiento de las matemáticas. Universidad Complutense de Madrid.

Esquivel, E. C., Araya, R. G., \& Sánchez, M. C. (2008). Creencias de los estudiantes en los procesos de aprendizaje de las matemáticas. Cuadernos de investigación y formación en educación Matemática, (4). 
Fernándes, I. (2009). Fortalezas y dificultades de los estudiantes de básica según sus desempeños en saber. Recuperado de: https://www. mineducacion.gov.co/1621/article-241773. html

Fernández Cézar, R., Prada Núñez, R., Hernández Suárez, C. A., \& Ramirez Leal, P (2018). Dominio afectivo y prácticas pedagógicas de docentes de Matemáticas.

García Sánchez, J., Aguilera Terrats, J. R., \& Castillo Rosas, A. (2011). Guía técnica para la construcción de escalas de actitud.

Gil Ignacio, N., Guerrero Barona, E., \& Blanco Nieto, L. (2006). El dominio afectivo en el aprendizaje de las Matemáticas.

Gil, N., Blanco, L. J., \& Guerrero, E. (2005). El dominio afectivo en el aprendizaje de las matemáticas. Una revisión de sus descriptores básicos. Revista iberoamericana de educación matemática, 2(1), 15-32.

Hernández, R., Fernández, C \& Baptista, M. (2010). Metodología de la Investigación, 5ta. Ed., México, DF, México: MacGraw-Hill.

Hernández, R., Fernández, C \& Baptista, P. (2003). Metodología de la investigación (Vol. 3). México: McGraw-Hill.

Hernández-Suarez, C. A., Prada-Núñez, R., \& Ramírez-Leal, P. (2017). Obstáculos epistemológicos sobre los conceptos de límite y continuidad en cursos de cálculo diferencial en programas de ingeniería. Revista Perspectivas, 2(2), 73-83.

Hidalgo Alonso, S., Maroto Sáez, A., \& Palacios Picos, A. (2005). El perfil emocional matemático como predictor de rechazo escolar: relación con las destrezas y los conocimientos desde una perspectiva evolutiva. Educación matemática, 17(2).

Martínez Padrón, O. J. (2005). Dominio afectivo en educación matemática. Paradigma, 26(2), 7-34.

Ministerio de E d u c a c i ó $n$ Nacional. (2011). Decreto 1290 de 2009. Recuperadodehttp://www.mineducacion. gov.co/1621/articles- 187765_archivo_pdf_ decreto_1290.pdf

Noguera, S. D., Ramírez, W. D., \& Martínez, J. L. D. (2016). Correlación de las actitudes y el rendimiento académico en la asignatura de matemáticas. MATUA, 3(1).

Padrón, O. J. M. (2008). Actitudes hacia la matemática. Sapiens. Revista Universitaria de Investigación, 9(1), 237-256.

Penagos, M., Mariño, L. F., \& Hernández, R. V. (2017). Pensamiento matemático elemental y avanzado como actividad humana en permanente evolución. Revista Perspectivas, 2(1), 105-116.

Rosal, A. A. S. (2010). Estrategias didácticas para el aprendizaje de los contenidos de trigonometría empleando las TICs. EDUTEC. Revista electrónica de tecnología educativa, (31), 130.

Velásquez-Luna, S. J., Celis-Gutiérrez, J. L., \& Hernández-Suárez, C. A. (2017). Evaluación contextualizada como estrategia docente para potenciar el desarrollo de competencias matemáticas en Pruebas Saber. Eco matemático, 8(s1), 33-37. 